\title{
DISTRIBUIÇÃO DO SISTEMA RADICULAR DE ÁRVORES DE ACÁCIA- NEGRA ORIUNDAS DE MUDAS PRODUZIDAS EM DIFERENTES RECIPIENTES ${ }^{1}$
}

Aline Fagote Paulino ${ }^{2}$, Cristiane de Conti Medina ${ }^{3}$, Carmen Silvia Vieira Janeiro Neves ${ }^{3}$, Mateus Carvalho Basílio de Azevedo ${ }^{4}$, Antonio Rioyei Higa ${ }^{5}$ e Augusto Simon ${ }^{6}$

\begin{abstract}
RESUMO - O objetivo deste trabalho foi estudar a distribuição do sistema radicular de árvores de acácia-negra (Acacia mearnsii) com 3 anos de idade, provenientes de mudas formadas em diferentes recipientes e instaladas em um Argissolo no Rio Grande do Sul. Os recipientes utilizados foram: laminado, tubete redondo e bandeja de isopor. As avaliações da distribuição das raízes foram feitas pelo método da parede do perfil até $1 \mathrm{~m}$ de profundidade, na linha e na entrelinha de plantio. Foram feitas, também, análises físicas do solo: densidade, porosidade total e distribuição de poros. O recipiente usado na formação da muda influenciou o crescimento do sistema radicular da planta no campo. O laminado de madeira apresentou-se superior ao tubete quanto ao comprimento de raízes nas linhas de plantio. As raízes cresceram melhor nas linhas de plantio, onde as condições de densidade e porosidade do solo se encontravam mais adequadas.
\end{abstract}

Palavras-chave: Acacia mearnsii, mudas, raízes, recipiente e solo.

\section{INFLUENCE OF SEEDLING PRODUCTION METHOD ON THE ROOT SYSTEM DISTRIBUTION OF BLACK WATTLE TREES}

\begin{abstract}
The purpose of this study was to evaluate the root system distribution of three-year-old black wattle (Acacia mearnsii) trees. The plants were originated from seedlings grown in different containers and planted in an Argisol in the state of Rio Grande do Sul, Brazil. The containers used for seedling production were: wood laminated pots, round plastic tubettes and styrofoam trays. The evaluations were carried out using the trench profile method up to $1 \mathrm{~m}$ depth on tree rows and inter-rows. The following soil analyses were also carried out: bulk density, total porosity and pore distribution. The type of container used influenced the development of the root system of trees on the field. Wood laminated pots were better than the round tubettes for root length in the tree rows. The roots developed better on the tree rows where soil density and porosity conditions were more adequate.
\end{abstract}

Key words: Acacia mearnsii, seedlings, root, recipient, and soil.

1 Recebido para publicação em 17.7.2002.

Aceito para publicação em 9.9.2003.

2 Eng ${ }^{\mathrm{a}}$-Agr ${ }^{\mathrm{a}}$., aluna de Mestrado em Agronomia, Universidade Estadual de Londrina, bolsista da CAPES. ${ }^{3}$ Eng $^{\mathrm{a}}$--Agr ${ }^{\mathrm{a}}$., Prof ${ }^{\mathrm{a}}$. Dra ${ }^{\text {a }}$ do Departamento de Agronomia, Universidade Estadual de Londrina, Caixa Postal 6001, 86051-990, <medina@uel.br>; ${ }^{4}$ Aluno de graduação em Agronomia, Universidade Estadual de Londrina. ${ }^{5}$ Eng. Florestal, Ph.D., Prof. do Departamento de Silvicultura e Manejo, Universidade Federal do Paraná, Rua Bom Jesus, 650, 80035-010 Curitiba-PR. ${ }^{6}$ Eng. Florestal, Tanagro S.A. Rua Weibull, 199, 95780-000 Montenegro-RS. 


\section{INTRODUÇÃO}

A acácia-negra (Acacia mearnsii De Wild.) é uma leguminosa que atinge de 6 a $20 \mathrm{~m}$ de altura e seu tronco, de 10 a $60 \mathrm{~cm}$ de diâmetro. Originada no sudoeste da Austrália e da Tasmânia, a principal finalidade de seu cultivo, no Brasil, é a extração do tanino da sua casca, que é utilizado na indústria de couro (Duke, 1981). A espécie é economicamente importante no Rio Grande do Sul, onde existem cerca de 140.000 ha plantados (Tonietto \& Stein, 1997). Porém, os fortes ventos que vêm ocorrendo nesses últimos anos têm causado o tombamento de árvores, fato que vem preocupando os produtores e comprometendo a produção na região (Medina et al., 1999).

Esses tombamentos ocasionados pelos ventos indicam que o sistema radicular é muito superficial. Dentre os fatores que podem influenciar o crescimento do sistema radicular destacam-se o recipiente em que as mudas cresceram e as condições físicas do solo. Barroso et al. (2000) verificaram que o recipiente de formação das mudas influencia o desenvolvimento do sistema radicular, devendo ser ressaltado que recipientes com paredes rígidas causam deformações nas raízes, o que pode comprometer o desenvolvimento inicial das mudas.

O recipiente onde a muda é produzida deve evitar que as raízes sofram deformações e enovelamento (Barroso et al., 2000; Leles et al., 2000). Segundo Reis et al. (1989), caso haja restrições ao desenvolvimento radicular, a má formação inicial das raízes pode persistir após o plantio, prejudicando o desenvolvimento das plantas no campo.

Reis et al. (1989) e Dedecek \& Gava (1997), relacionando as características físicas do solo ao crescimento radicular de plantas de eucalipto, verificaram que a compactação do solo altera a sua estrutura, dificultando o crescimento e a distribuição de raízes. Malinovski (1996) e Camargo \& Alleoni (1997) verificaram que a compactação do solo influencia negativamente o crescimento das raízes das plantas. A caracterização da distribuição do sistema radicular no perfil do solo é importante para a definição e a tomada de decisões a respeito de importantes práticas de manejo florestal (Mello et al., 1997).

O objetivo deste trabalho foi estudar a distribuição do sistema radicular de árvores de acácia-negra provenientes de mudas formadas em diferentes recipientes e plantadas em um Argissolo do Rio Grande do Sul.

R. Árvore, Viçosa-MG, v.27, n.5, p.605-610, 2003

\section{MATERIAL E MÉTODOS}

O experimento foi instalado em um Argissolo, na fazenda Ouro Verde, no município de Cristal-RS, em área experimental da TANAC S.A. Antes do plantio de acácianegra, a área estava ocupada por pastagem, onde o gado era criado no sistema extensivo. Para o preparo do solo, foram feitas duas subsolagens, seguidas da abertura de sulco, para efetuar o plantio e a adubação. Foi adotado o espaçamento de 3,0 x 1,5 m. A avaliação do sistema radicular foi realizada quando as plantas atingiram 3 anos de idade. Os dados das análises granulométricas do solo mostraram $600 \mathrm{~g} / \mathrm{kg}$ de areia grossa, $140 \mathrm{~g} / \mathrm{kg}$ de areia fina, $150 \mathrm{~g} / \mathrm{kg}$ de silte e $110 \mathrm{~g} / \mathrm{kg}$ de argila, na profundidade de 0 a $40 \mathrm{~cm}$, e $380 \mathrm{~g} / \mathrm{kg}$ de areia grossa, $120 \mathrm{~g} / \mathrm{kg}$ de areia fina, $230 \mathrm{~g} / \mathrm{kg}$ de silte e $270 \mathrm{~g} / \mathrm{kg}$ de argila, na profundidade de 40 a $80 \mathrm{~cm}$.

Foram testados três tipos de recipientes: laminado de madeira com volume de $353,43 \mathrm{~cm}^{3}$; tubete de plástico redondo com $50 \mathrm{~cm}^{3}$; e bandeja de isopor com células piramidais de $180 \mathrm{~cm}^{3}$. Os recipientes foram preenchidos com o substrato comercial Plantmax ${ }^{\circledR}$, constituído de uma mistura de vermiculita, casca de pinus compostada e fertilizantes.

Para avaliação do sistema radicular, foram selecionadas plantas que representavam a média do crescimento dentro de cada parcela. Foram, então, abertas quatro trincheiras em cada tratamento (recipientes) a partir do tronco da planta, com dimensões de $0,75 \mathrm{~m}$ de comprimento por 1,0 $\mathrm{m}$ de profundidade na linha, e de 1,5 $\mathrm{m}$ de comprimento por 1,0 $\mathrm{m}$ de profundidade na entrelinha de plantio, de acordo com a metodologia da parede do perfil ou trincheira (Böhm, 1979). O perfil foi submetido a um jato de água para expor as raízes. Em seguida, estas foram individualmente pintadas com tinta esmalte branca, para maior contraste com o solo, o que facilitou as avaliações posteriores. Ajustou-se uma tela de malha de 25 x $25 \mathrm{~cm}$ ao perfil, e os quadros foram fotografados nas profundidades de 0-25, 25-50, 50-75 e 75-100 cm na linha e na entrelinha, obtendo-se, assim, 36 imagens por árvore. Estas imagens foram digitalizadas e analisadas pelo programa SIARCS $®$, obtendo-se o comprimento de raízes (cm), de acordo com o descrito por Jorge et al. (1996).

As amostras de solo foram coletadas em todas as trincheiras nas linhas de plantio (L) e em duas distâncias do tronco nas entrelinhas, tendo EA representado a distância de $33 \mathrm{~cm}$ e EB a distância de $66 \mathrm{~cm}$, e nas profundidades de 0-10, 10-20, 20-40 e 40-60 cm. 
As amostras de solo foram coletadas com o auxílio de anéis e enviadas para o laboratório, onde foram determinadas a densidade do solo, a porosidade total e as macro e microporosidades, pelo método da mesa de tensão (Embrapa, 1997).

O delineamento experimental utilizado foi o inteiramente casualizado, com três tratamentos e quatro repetições. Os dados dos comprimentos totais e de porcentagem de raízes por perfil foram avaliados pela análise de variância. As interações entre tratamento x profundidade e tratamento $\mathrm{x}$ distância do tronco foram avaliadas por meio de parcelas subdivididas. Os resultados foram submetidos à análise de variância e complementados pelo teste de Tukey, a 5\% de probabilidade.

\section{RESULTADOS E DISCUSSÃO}

Não houve diferença significativa para o comprimento total de raízes por planta (Quadro 1) entre os tratamentos na entrelinha e na entrelinha + linha, porém foi observada diferença na linha de plantio. $O$ tratamento tubete apresentou valores significativamente inferiores aos do tratamento laminado de madeira.

A superioridade do laminado de madeira pode ser explicada pelo seu maior volume para o desenvolvimento do sistema radicular. De acordo com Leles et al. (2000), recipientes que forneçam maior espaço para o crescimento das raízes são mais adequados. Barroso et al. (2000) verificaram que mudas de Eucalyptus spp. produzidas em tubetes apresentaram raiz pivotante indefinida e bifurcada. Observaram, também, raízes laterais primárias finas e pouco ramificadas, o que dificulta a exploração adequada do solo. Desta forma, no presente ensaio, o recipiente de laminado favoreceu a melhor formação inicial das raízes, que se refletiu na melhor distribuição do sistema radicular depois do plantio no campo.

Considerando a densidade de raízes (Quadro 1), os valores encontrados nas entrelinhas foram inferiores aos encontrados nas linhas de plantio em todos os tratamentos, havendo maior concentração de raízes nas linhas do que nas entrelinhas. Tal resultado pode ser explicado pelo fato de as densidades do solo nas linhas serem menores do que as densidades encontradas nas entrelinhas, enquanto a porosidade total teve comportamento inverso para todas as profundidades (Quadro 5). Segundo Malinovski (1996) e Reis et al. (1989), a densidade do solo influencia diretamente o crescimento do sistema radicular. Borges et al. (1986), estudando o sistema radicular de Eucalyptus spp. em vasos, constataram que a presença de uma camada compactada levou as plantas a acumularem maior quantidade de raízes na camada superficial. Observaram, ainda, que o crescimento de raízes foi menor onde as densidades do solo eram elevadas. Outra explicação para as raízes terem se desenvolvido melhor nas linhas é o fato de a adubação ter sido realizada no sulco de plantio, favorecendo o crescimento radicular nesse local.

Analisando as porcentagens de raízes por tratamento e em função da profundidade (Quadro 2), verifica-se que a camada de 0 a $25 \mathrm{~cm}$ foi sempre significativamente superior às demais, apresentando 62,95, 59,46 e 56,27\%, para tubete, laminado e bandeja de isopor, respectivamente. As porcentagens de raízes de $75 \mathrm{a} 100 \mathrm{~cm}$ foram significativamente inferiores às camadas de $0 \mathrm{a} 25$ e 25 a $50 \mathrm{~cm}$, para os três tipos de recipientes. Este fato pode

Quadro 1 - Comprimentos totais $(\mathrm{cm})$ de raízes de acácia-negra aos três anos de idade nas linhas, entrelinhas e linhas + entrelinhas de plantio e densidade de raízes nas linhas e entrelinhas de plantio $\left(\mathrm{cm} / \mathrm{cm}^{2}\right)$, em função de tipos de recipientes usados no viveiro. Cristal-RS, 1999

Table 1 - Total root length $(\mathrm{cm})$ and root density $\left(\mathrm{cm} / \mathrm{cm}^{2}\right)$ of three-year-old black wattle trees on field rows, inter-rows, and rows + inter-rows, based on type of nursery recipients used Cristal-RS, 1999

\begin{tabular}{|l|c|c|c|c|c|}
\hline \multirow{2}{*}{ Tratamento } & \multicolumn{3}{|c|}{ Comprimento de Raízes $(\mathrm{cm})$} & \multicolumn{2}{c|}{ Densidade de Raízes $\left(\mathrm{cm} / \mathrm{cm}^{2}\right)$} \\
\cline { 2 - 6 } & Entrelinha & Linha & Entrelinha+Linha & Entrelinha & Linha \\
\hline Laminado & $2.323,5 \mathrm{~A}$ & $2.048,8 \mathrm{~A}$ & $4.371,8 \mathrm{~A}$ & $15,5 \mathrm{~b}$ & $27,3 \mathrm{a}$ \\
\hline Tubete & $1.371,8 \mathrm{~A}$ & $917,5 \mathrm{~B}$ & $2.289,2 \mathrm{~A}$ & $9,4 \mathrm{~b}$ & $12,2 \mathrm{a}$ \\
\hline Bandeja & $1.633,3 \mathrm{~A}$ & $1.179,2 \mathrm{AB}$ & $2.812,5 \mathrm{~A}$ & $10,9 \mathrm{~b}$ & $15,7 \mathrm{a}$ \\
\hline \multicolumn{1}{c|}{$\mathrm{CV}(\%)$} & 38,14 & 30,41 & 30,57 & \multicolumn{2}{c}{32,97} \\
\hline
\end{tabular}

Letras diferentes, minúsculas nas linhas e maiúsculas nas colunas, indicam diferença significativa (Tukey, $\mathrm{p}<0,05$ ). 
ter ocorrido em função da textura do solo, pois as análises granulométricas indicaram a presença de uma concentração maior de argila na camada de 40 a $80 \mathrm{~cm}$ em relação à camada superficial, o que ocorre naturalmente em Argissolos e forma uma barreira natural à penetração de raízes.

Quadro 2 - Porcentagem (\%) de raízes de acácia-negra aos 3 anos de idade, em diferentes profundidades do solo, em função de recipientes usados no viveiro. Cristal-RS, 1999

Table 2 - Root percent (\%) of three-year-old black wattle trees in different soil depths, based on type of nursery recipients used. Cristal-RS, 1999

\begin{tabular}{|c|c|c|c|}
\hline \multirow{2}{*}{$\begin{array}{c}\text { Profundidade } \\
(\mathrm{cm})\end{array}$} & \multicolumn{3}{|c|}{ Tratamento } \\
\cline { 2 - 4 } & Laminado & Tubete & $\begin{array}{c}\text { Bandeja de } \\
\text { isopor }\end{array}$ \\
\hline $0-25$ & $59,46 \mathrm{~A}$ & $62,95 \mathrm{~A}$ & $56,27 \mathrm{~A}$ \\
\hline $25-50$ & $21,48 \mathrm{~B}$ & $22,18 \mathrm{~B}$ & $22,98 \mathrm{~B}$ \\
\hline $50-75$ & $11,67 \mathrm{BC}$ & $9,48 \mathrm{C}$ & $12,72 \mathrm{BC}$ \\
\hline $75-100$ & $7,39 \mathrm{C}$ & $5,39 \mathrm{C}$ & $8,03 \mathrm{C}$ \\
\hline $\mathrm{CV}(\%)$ & 12,99 & 10,89 & 12,82 \\
\hline
\end{tabular}

Letras diferentes maiúsculas nas colunas indicam diferença significativa (Tukey, $\mathrm{p}<0,05$ ).

Em florestas com árvores que podem chegar a $20 \mathrm{~m}$ de altura, um sistema radicular superficial como o observado neste estudo, com mais de $56 \%$ de raízes na camada de 0 a $25 \mathrm{~cm}$ para os três tratamentos, pode comprometer a produtividade do povoamento, pois as árvores poderão ter dificuldades em absorver água se ocorrer estresse hídrico e há riscos de tombamento em caso de ventos fortes, por não haver boa sustentação da planta.

Os resultados das análises estatísticas com parcelas subdivididas, considerando-se tratamentos e as profundidades do solo, ou as distâncias do tronco, encontramse nos Quadros 3 e 4. As interações entre tratamento x profundidade e tratamento x distância não foram significativas. Verificou-se que os comprimentos de raízes diminuíram nas linhas e entrelinhas à medida que houve aumento da profundidade para os três tipos de recipientes (Quadro 3), o que confirma os resultados encontrados por diversos autores em culturas perenes (Mello et al., 1997; Neves et al., 1998, 2001). Na linha de plantio (Quadro 3), o comprimento de raízes na profundidade de 0 a $25 \mathrm{~cm}$ foi significativamente superior ao das demais camadas.

Quando são comparadas as quantidades de raízes por recipiente e por profundidade (Quadro 3), observase o mesmo resultado obtido para os totais de raízes (Quadro 1), pois não houve diferença significativa nas entrelinhas; já na linha o laminado proporcionou maior comprimento de raízes, diferenciando-se significativamente do tratamento tubete, provavelmente pelas melhores condições físicas (Quadro 5) e químicas (em função da adubação em sulco).

Quadro 3 - Comprimento de raízes (cm) de acácia-negra aos 3 anos de idade na entrelinha e linha de plantio em diferentes profundidades, em função de tipos de recipientes usados no viveiro. Cristal-RS, 1999

Table 3 - Root length (cm) of black three-year-old wattles on field inter-rows and rows in different depths, based on type of nursery recipients used. Cristal-RS, 1999

\begin{tabular}{|c|c|c|c|c|c|c|}
\hline \multirow{2}{*}{ Tratamento } & \multirow{2}{*}{ Posição } & \multicolumn{4}{|c|}{ Profundidade $(\mathrm{cm})$} & \multirow{2}{*}{ Média } \\
\hline & & $0-25$ & $25-50$ & $50-75$ & $75-100$ & \\
\hline Laminado & \multirow{3}{*}{ Entrelinha } & $1.402,2$ & 492,9 & 255,4 & 172,9 & $580,9 \mathrm{~A}$ \\
\hline Tubete & & 826,0 & 311,9 & 145,4 & 88,4 & $342,9 \mathrm{~A}$ \\
\hline Bandeja & & 927,3 & 362,5 & 195,9 & 147,5 & $480,3 \mathrm{~A}$ \\
\hline Média & & $1.051,8 \mathrm{~A}$ & $389,1 \mathrm{~B}$ & $1.98,9 \mathrm{BC}$ & $136,3 \mathrm{C}$ & \\
\hline Laminado & \multirow{3}{*}{ Linha } & $1.273,1$ & 451,3 & 179,3 & 144,9 & $512,2 \mathrm{~A}$ \\
\hline Tubete & & 601,0 & 201,4 & 79,0 & 35,9 & $229,3 \mathrm{~B}$ \\
\hline Bandeja & & 636,2 & 306,4 & 154,9 & 81,6 & $2.94,8 \mathrm{AB}$ \\
\hline Média & & $836,8 \mathrm{~A}$ & $319,7 \mathrm{~B}$ & $1.37,8 \mathrm{~B}$ & $87,5 \mathrm{~B}$ & \\
\hline \multicolumn{4}{|c|}{$\begin{array}{l}\text { CV }(\%) \text { Tratamento entrelinha }=77,71 \\
\text { CV }(\%) \text { Profundidade entrelinha }=43,63\end{array}$} & \multicolumn{3}{|c|}{$\begin{array}{l}\mathrm{CV}(\%) \text { tratamento linha }=65,95 \\
\mathrm{CV}(\%) \text { profundidade linha }=59,76\end{array}$} \\
\hline
\end{tabular}

Letras diferentes, minúsculas nas linhas e maiúsculas nas colunas, indicam diferença significativa $($ Tukey, $\mathrm{p}<0,05)$. 
Quadro 4 - Comprimento de raízes (cm) de acácia-negra aos 3 anos de idade na entrelinha e linha de plantio em diferentes distâncias do tronco, em função de tipos de recipientes usados no viveiro. Cristal-RS, 1999

Table 4 - Root length (cm) of three-year-old black wattle trees on field inter-rows and rows in different distances from the trunk, based on type of nursery recipient used. Cristal-RS, 1999

\begin{tabular}{|c|c|c|c|c|c|c|c|c|}
\hline \multirow{2}{*}{ Tratamento } & \multirow{2}{*}{ Posição } & \multicolumn{6}{|c|}{ Distância $(\mathrm{cm})$} & \multirow{2}{*}{ Média } \\
\hline & & $0-25$ & $25-50$ & $50-75$ & $75-100$ & $100-125$ & $125-150$ & \\
\hline Laminado & \multirow{3}{*}{ Entrelinha } & 561,8 & 352,3 & 341,3 & 333,9 & 363,4 & 370,7 & $387,3 \mathrm{~A}$ \\
\hline Tubete & & 294,3 & 463,2 & 251,5 & 207,8 & 197,0 & 127,7 & $256,9 \mathrm{~A}$ \\
\hline Bandeja & & 299,3 & 308,7 & 289,5 & 199,9 & 323,2 & 203,6 & $255,4 \mathrm{~A}$ \\
\hline Média & & $418,5 \mathrm{a}$ & $374,7 \mathrm{ab}$ & $294,1 \mathrm{AB}$ & $247,2 \mathrm{AB}$ & $264,2 \mathrm{~B}$ & $234,0 \mathrm{~B}$ & \\
\hline Laminado & \multirow{3}{*}{ Linha } & 667,8 & 717,4 & 663,1 & -- & -- & -- & $682,8 \mathrm{~A}$ \\
\hline Tubete & & 286,5 & 305,9 & 325,0 & -- & -- & -- & $305,8 \mathrm{~B}$ \\
\hline Bandeja & & 441,5 & 417,6 & 321,8 & -- & -- & -- & $393,63 \mathrm{AB}$ \\
\hline Média & & $465,3 \mathrm{a}$ & $480,3 \mathrm{a}$ & $436,6 \mathrm{~A}$ & -- & -- & -- & \\
\hline \multicolumn{5}{|c|}{$\begin{array}{l}\text { CV (\%) Tratamento entrelinha }=73,43 \\
\text { CV (\%) Distância entrelinha }=39,74\end{array}$} & \multicolumn{4}{|c|}{$\begin{array}{l}\text { CV }(\%) \text { tratamento linha }=52,97 \\
\text { CV }(\%) \text { distância linha }=35,05\end{array}$} \\
\hline
\end{tabular}

Letras diferentes, minúsculas nas linhas e maiúsculas nas colunas, indicam diferença significativa (Tukey, p<0,05).

Quadro 5 - Densidade do solo (DS), porosidade total (PT), macro e microporosidades do solo cultivado com acácianegra na linha e na entrelinha de plantio, nas profundidades $0-10,10-20,20-40$ e $40-60 \mathrm{~cm}$. Cristal-RS, 1999

Table 5 - Soil bulk density (DS), soil total porosity (TP), macro and microporosities of soil cultivated with black wattle on tree rows and inter-rows, in depths of 0-10, 10-20, 20-40 and 40-60 cm. Cristal- RS, 1999

\begin{tabular}{|c|c|c|c|c|}
\hline $\begin{array}{c}\text { Amostra e } \\
\text { Profundidade } \\
(\mathrm{cm})\end{array}$ & $\mathrm{DS}$ & $\mathrm{PT}$ & $\begin{array}{c}\text { Macroporo- } \\
\text { sidade }\end{array}$ & $\begin{array}{c}\text { Microporo- } \\
\text { sidade }\end{array}$ \\
\cline { 3 - 5 }$\left(\mathrm{kg} / \mathrm{dm}^{3}\right)$ & \multicolumn{3}{|c|}{$\left(\mathrm{dm}^{3} / \mathrm{dm}^{3}\right)$} \\
\hline $\mathrm{L}(0-10)$ & 1,41 & 0,047 & 0,027 & 0,020 \\
\hline $\mathrm{L}(10-20)$ & 1,47 & 0,045 & 0,019 & 0,026 \\
\hline $\mathrm{L}(20-40)$ & 1,41 & 0,047 & 0,016 & 0,031 \\
\hline $\mathrm{L}(40-60)$ & 1,34 & 0,049 & 0,024 & 0,025 \\
\hline EA (0-10) & 1,51 & 0,043 & 0,022 & 0,021 \\
\hline EA (10-20) & 1,53 & 0,042 & 0,018 & 0,024 \\
\hline EA (20-40) & 1,53 & 0,042 & 0,014 & 0,028 \\
\hline EA (40-60) & 1,53 & 0,042 & 0,021 & 0,021 \\
\hline EB $(0-10)$ & 1,54 & 0,042 & 0,020 & 0,022 \\
\hline EB (10-20) & 1,58 & 0,040 & 0,013 & 0,027 \\
\hline EB (20-40) & 1,53 & 0,042 & 0,015 & 0,027 \\
\hline EB (40-60) & 1,47 & 0,045 & 0,017 & 0,028 \\
\hline
\end{tabular}

$\mathrm{L}=$ linha de plantio, $\mathrm{EA}=$ entrelinha de plantio a $33 \mathrm{~cm}$ do tronco e $\mathrm{EB}=$ entrelinha de plantio a $66 \mathrm{~cm}$ do tronco.

Quanto ao comprimento de raízes em diferentes distâncias do tronco, observa-se que na entrelinha de plantio (Quadro 4) não houve diferença significativa entre os diferentes recipientes, mas foi observada diferença na linha, tendo o laminado sido superior à bandeja de isopor. Quando as distâncias são comparadas, constata-se que a de 0 a $25 \mathrm{~cm}$ apresentou-se superior, diferenciando significativamente das distâncias de 100 a 125 e 125 a $150 \mathrm{~cm}$ do tronco, o que indica que há uma concentração de raízes até $100 \mathrm{~cm}$, a partir do tronco, na entrelinha.

De modo geral, pôde-se observar que, em função das características do solo da região, as plantas tiveram dificuldades para o crescimento das raízes, porém estas dificuldades foram amenizadas nas linhas de plantio pelo preparo do solo. Além disto, as plantas produzidas em laminados, que tiveram melhores condições de crescimento de raízes na fase de muda, superaram melhor estas dificuldades.

\section{CONCLUSÕES}

Pelos resultados obtidos neste trabalho, pode-se concluir que:

O recipiente usado na formação da mudas de acácianegra influencia o crescimento radicular das plantas após o plantio.

O comprimento de raízes de acácia-negra nas linhas de plantio é maior quando as mudas são produzidas em recipiente de laminado de madeira do que em tubete.

As raízes de acácia-negra crescem melhor nas linhas de plantio do que nas entrelinhas.

R. Árvore, Viçosa-MG, v.27, n.5, p.605-610, 2003 


\section{REFERÊNCIAS BIBLIOGRÁFICAS}

BARROSO, D. G. et al. Efeitos do recipiente sobre o desempenho pós-plantio de Eucalyptus camaldulensis Dehnh. e E. urophylla S.T. Blake. Revista Árvore, v. 24, n. 3, p. 291-296, 2000.

BÖHM, W. Methods of studing root sistems. New York: Spring-Verlag, 1979. $185 \mathrm{p}$.

BORGES, E. N. Resposta de mudas de Eucalyptus a camadas compactadas de solo. Revista Árvore, v. 10, n. 2, p. $181-195,1986$.

CAMARGO, O. A.; ALLEONI, L. R. F. Compactação do solo e desenvolvimento das plantas. Piracicaba: Degaspar, 1997. $132 \mathrm{p}$.

DEDECEK, R. A.; GAVA, J. L. Compactação do solo pela colheita de eucalipto: sua avaliação e efeito na produtividade da rebrota. In: CONFERENCE ON SILVICULTURE AND IMPROVEMENT OF EUCALYPTS, Salvador, 1997. Proceeding... Salvador: IUFRO, 1997. p. 63-68.

DUKE, J. A. Handbook of legumes of world economic importance. New York: Plenum Press, 1981. 345 p.

EMPRESA BRASILEIRA DE PESQUISA AGROPECUÁRIA - EMBRAPA. Manual de métodos de análise de solo. 2.ed. Rio de Janeiro: SNLCS, 1997. 212 p.

JORGE, L. A. C. et al. Aquisição de imagens de raízes. In: JORGE, L. A. C. (Ed.) Recomendações práticas para aquisição de imagens digitais analisadas através do SIARCS. São Carlos: EMBRAPA - Instrumentação Agropecuária, 1996. 48 p. Cd-Rom.
LELES, P. S. S. et al. Qualidade de mudas de Eucalyptus spp. produzidas em blocos prensados e em tubetes. Revista Árvore, Viçosa, v. 24, n. 1, p. 13-20, 2000.

MALINOVSKI, J. R. Compactação dos solos usados para fins florestais. In: CONGRESSO LATINO AMERICANO DE CIÊNCIA DO SOLO, 1996, Águas de Lindóia.

Resumos expandidos... Águas de Lindóia: 1996. CD-Rom.

MEDINA, C. C.; NEVES, C. S. V. J.; HIGA, A. R. Arquitetura do sistema radicular de acácia negra (Acacia mearnsii) no Rio Grande do Sul. In: WORKSHOP SOBRE SISTEMA RADICULAR: METODOLOGIAS E ESTUDO DE CASOS, 1999, Aracaju. Anais... Aracaju: 1999. p. $245-252$.

MELLO, S. L. M. et al. Características do sistema radicular em povoamentos de eucaliptos propagados por sementes e estacas. In: CONFERENCE ON SILVICULTURE AND IMPROVEMENT OF EUCALYPTS, 1997, Salvador. Proceedings... Salvador: IUFRO, 1997. p. 54-62.

NEVES, C. S. V. J. et al. Distribuição do sistema radicular de cultivares de aceroleira. Revista Brasileira de Fruticultura, v. 23, n. 1, p. 112-115, 2001.

NEVES, C. S. V. J. et al. Efeito do manejo do solo no sistema radicular de tangerina "Poncã" enxertada sobre limoeiro "Cravo" em latossolo roxo. Revista Brasileira de Fruticultura, v. 20, n. 2, p. 246-253, 1998.

TONIETTO, L.; STEIN, P. P. Silvicultura da acácia-negra (Acacia mearnsii DE Wild.) no Brasil. Florestal Estatístico, v. 4, n. 12, p. 11-16, 1996/1997.

REIS, G. G. et al. Crescimento de Eucalyptus camaldulensis, E. grandis e E. cloeziana sob diferentes níveis de restrição radicular. Revista Árvore, v. 13, n. 1, p. 1-18, 1989. 